\title{
Nebular condensation of different stellar compositions and its influence on planetary chemistry
}

\section{Other Conference Item}

\section{Author(s):}

Wang, Haiyang (1); Sossi, Paolo A.; Quanz, Sascha Patrick (1)

Publication date:

2020-09

Permanent link:

https://doi.org/10.3929/ethz-b-000466668

Rights / license:

Creative Commons Attribution 4.0 International

Originally published in:

EPSC Abstracts 14, https://doi.org/10.5194/epsc2020-874 


\title{
Nebular condensation of different stellar compositions and its influence on planetary chemistry
}

\author{
Haiyang Wang ${ }^{1}$, Paolo Sossi ${ }^{2}$, and Sascha Quanz ${ }^{1}$ \\ ${ }^{1}$ Institute for Particle Physics and Astrophysics, ETH Zürich, CH-8093, Zürich, Switzerland (haiwang@phys.ethz.ch; \\ sascha.quanz@phys.ethz.ch) \\ ${ }^{2}$ Institute of Geochemistry and Petrology, ETH Zürich, CH-8092, Zürich, Switzerland (paolo.sossi@erdw.ethz.ch)
}

The volatility of an element is defined by its $50 \%$ condensation temperature $\left(\mathrm{T}_{\mathrm{c}}^{50}\right)$ from a canonical nebular gas of Solar composition at $10^{-4}$ bar $[1,2]$. However, the variability in metallicity and metal/oxygen ratios of extrasolar systems inferred from the spectroscopic measurements of their parent stars $[3,4]$ implies that the identity, abundance and sequence of condensation may deviate from that of our solar system. As such, planets formed at similar heliocentric distances may be expected to have distinct compositions from those of the terrestrial planets in our solar system. Here we investigate the degree to which nebular composition influences the condensation process by taking nine sets of stellar compositions with variable metallicities that span the range from -0.4 to +0.4 dex and performing Gibbs free energy minimisation calculations with FactSage, including treatment of mineral solid-solutions, over the temperature range $1723 \mathrm{~K}$ to $473 \mathrm{~K}$. We find that, although the general order of condensation is similar, absolute values of $\mathrm{T}_{\mathrm{c}}^{50}$ are shifted to higher temperatures at higher dex, where $\mathrm{T}_{\mathrm{c}}{ }^{50}(\mathrm{~S})$, in particular, increases relative to those of other elements. Condensing nebulae with high metallicities (and also high metal/oxygen ratios) also exhibit the following features: (i) the appearance of reduced assemblages (e.g. CaS oldhamite, forsterite-rich olivine and graphite) in the condensates, (ii) increased fractions of oxygen (relative to its total abundance) locked in the silicate condensates, and (iii) lower $\mathrm{fO}_{2}$ in the gas phase. As a result, these characteristics will lead to significant differences in the chemistry of planetary building blocks, which are then accreted to form telluric planetary bodies.

\section{References}

[1] Lodders 2003. Ap] 591:1220-1247.

[2] Wood, B. J., Smythe, D. J., \& Harrison, T. 2019. Ame. Miner. 104:844-856.

[3] Buder, S., Asplund, M., Duong, L. et al. 2018. MNRAS 478:4513:4552.

[4] Delgado Mena, E., Moya, A., Adibekyan, V., et al. 2019. A\&A 624:A78. 